\title{
An odd effect: Lengthened reaction times for judgments about odd digits
}

\author{
TERENCE M. HINES \\ Pace University, Pleasantville, New York
}

\begin{abstract}
Eight experiments are reported that first establish and then explicate a serendipitous finding that judgments about whether digits are odd or even take longer for odd than for even digits. The slowing of judgments about odd digits is more pronounced when digit pairs or triples are used, but is still weakly present when a single digit must be classified. A similar effect is seen when judgments of nouns are based on whether the nouns are the names of living or dead objects. Nouns that name dead objects are judged more slowly than ones that name living objects. The concept "alive" is linguistically marked. Past research has shown that unmarked concepts are processed more rapidly than marked ones. The similarity in the pattern of results when digits and words are judged is used to argue that the slower judgments about odd digits are due to the fact that "odd" is a linguistically marked and "even" a linguistically unmarked concept.
\end{abstract}

The distinction between odd and even numbers is a fundamental one, both mathematically and psychologically. Shepard, Kilpatric, and Cunningham (1975) showed that the odd versus even distinction formed a major dimension of subjects' judgments of similarity among single digits when the similarity judgments were to be based on the digits as "abstract concepts." The importance of the oddness or evenness of the digits emerged regardless of whether the digits to be judged were presented as arabic numerals, rows of dots, or spoken words. In spite of the importance of the odd versus even distinction, as demonstrated by Shepard et al., differential processing of odd and even digits has apparently received no other explicit research attention.

Some research has, however, used the odd versus even distinction in a tangential fashion without directly examining processing differences between odd and even digits. Krueger (1986; Krueger \& Hallford, 1984) found that when the sum or product of a visually presented equation violates an "odd-even rule," subjects can apparently use the odd or even character of the sum or product to judge whether the equation is correct or incorrect. In addition, Sudevan and Taylor (1987) required subjects to make odd versus even judgments of single digits as part of a series of experiments aimed at examining the priming of mental operations. Sudevan (personal communication, August 7 , 1987) has reanalyzed the results of their Experiment 2 and found that odd digits were responded to $24 \mathrm{msec}$ ( $813 \mathrm{vs}$. $789 \mathrm{msec}$ ) slower than even digits. Experiment 1 of the present article unexpectedly obtained a similar finding and

Portions of the research reported here were supported by a Pace University Scholarly Research Fund grant (Hines \& Clauss, 1982). I thank Thomas H. Killion for suggesting Experiment 5. Reprint requests may be addressed to Terence Hines, Psychology Department, Pace University, Pleasantville, NY 10570-2799. led to the reported series of experiments on differential speed of response to odd and even digits.

\section{EXPERIMENT 1}

Experiment 1 was planned and run with no intention of examining differences between odd and even digits. Its goal was to investigate differences in the time course of encoding between young and normal elderly adults, and the results pertaining to that issue have been reported elsewhere (Hines, Poon, Cerella, \& Fozard, 1982).

The first hint of a difference between the odd and even digits came during subject debriefing, when several subjects spontaneously mentioned that odd-odd pairs had seemed more difficult to them. The data were then reanalyzed to see if they bore out the subjects' introspections. The original report of Hines et al. (1982) did not contain any analysis of the odd versus even effects reported here.

\section{Method}

Subjects. The subjects were 22 undergraduates from Boston-area colleges and universities between 18 and 27 years of age (mean = $21, S D=2.5$ ) and older persons between 56 and 79 years of age (mean $=71, S D=4.9$ ). The older subjects were recruited from homes for the elderly in the Boston area. The younger subjects were recruited through student employment offices of Boston-area colleges and universities. All subjects reported themselves to be in good health and traveled to the laboratory in downtown Boston unaided, usually via public transportation. The younger group had an average of 15 years of education; for the older group, this figure was 12 years. There were 11 females and 11 males in the younger group; there were 22 females and 5 males in the older group. Each subject was paid $\$ 15$ for participating. Before participating in the study reported here, the subjects had taken part in two unrelated short studies, both using reaction time (RT) as the dependent variable.

Apparatus and Stimuli. The subjects sat at a self-determined distance in front of a cathode-ray tube, which was used for stimulus presentation. The stimuli were all members of the computer's 
standard character set. Responses were made by pressing the appropriate one of two telegraph keys also directly in front of the subject. Control of all aspects of stimulus presentation and response recording was handled online by a PDP-8e computer.

Procedure. The stimuli were pairs of digits from the set 2, 3 , $4,5,6,7,8$, and 9 . Exact measurements of the size of the stimuli used are no longer available. The digits were presented centered about an asterisk that served as a warning signal.

The subject was to decide whether the two digits on each trial were the same or different. A trial began with presentation of the warning asterisk, which remained on the screen for $750 \mathrm{msec}$. Simultaneous with the offset of the warning asterisk, the first digit appeared. The second digit appeared at a stimulus-onset asynchrony (SOA) of $0,250,500,750$, or $1,000 \mathrm{msec}$ in a position to the right of the first digit. The 0 -msec SOA condition resulted in simultaneous presentation of the two digits. Both digits remained on the screen until the subject had responded. Following a 2,500 -msec intertrial interval, the warning signal for the next trial appeared. SOA on each trial was selected randomly from the five intervals. A rule-level match was required, for which only pairs of digits that were both odd (i.e., 7 and 3) or both even (i.e., 6 and 4) were judged as "same." Mixed odd-even pairs were to be regarded as "different." There were no physically identical digit pairs (i.e., 3, 3).

All subjects used the index finger on their left hand to press the left telegraph key to indicate "same" judgments. The index finger of the right hand and the right key were used for "different" responses. If an incorrect response was made, an audible "beep" sounded immediately following the response.

Three blocks of 80 trials were run. Rest periods were given between blocks. Twenty practice trials were given before the start of the first block. An equal number of same and different pairs appeared in each block.

\section{Results and Discussion}

Table 1 shows the mean RTs for young and old subjects to respond "same" to pairs of odd and pairs of even digits at each SOA. A three-way analysis of variance (ANOVA) (digit type $\times$ SOA $\times$ age) revealed three significant main effects. The elderly subjects responded more slowly than the young $[F(1,47)=32.52, p<.001]$, RT decreased as SOA increased $[F(4,188)=74.86, p<$ $.001]$, and odd digit pairs were responded to more slowly than even digit pairs $[F(1,47)=177.85, p<.001]$. Two of the two-way interactions were significant. That between age and digit type $[F(1,47)=21.39, p<.001]$ indicated that the relative amount of slowing for odd digit pairs was greater for the elderly than for the young subjects. The interaction between digit type and SOA $[F(4,188)=3.16$, $p<.016$ ] reflected the finding that $\mathrm{RT}$ decreased as a function of SOA at a somewhat slower rate for odd than for even digits. None of the other interactions reached significance.

A three-way ANOVA on the error-rate data revealed only one significant effect, with more errors being made on odd-odd $(8.8 \%)$ than on even-even pairs $(1.2 \%)$ $[F(1,47)=70.98, p<.001]$. No other main effects or interactions were significant. Data for different pairs were reported in Hines et al. (1982), to which the reader is referred.

Several of the significant main effects and interactions found in the ANOVA of RTs are easily explained. It is well known that older subjects have longer RTs than do younger subjects (Cerella, 1985). Furthermore, task difficulty almost always interacts with age, such that older subjects are slowed relatively more than younger subjects as task difficulty increases (Cerella, 1985). Thus, the interaction between digit type and age is not theoretically informative. The main effect of digit type, in the ANOVAS of both RTs and error rates, amply confirms the subjects' reports that the odd-odd pairs were more difficult than the even-even pairs.

Posner and Boies (1971) have termed the function relating RT to SOA the "encoding" function. The point along this function where $R T$ reaches a minimum indicates the time taken to encode the stimulus. Statistically, this is usually indicated by an interaction between the SOA variable and some stimulus or subject variable. Thus, in their original analysis, Hines et al. (1982) obtained a significant interaction between SOA and age, and argued that older subjects took longer to encode digits than did younger subjects. The original analysis included both same and different responses, unlike the present reanalysis, which examined only same responses. The data in Hines et al., when reanalyzed here, also show a significant interaction between SOA and the odd versus even variable, thus suggesting that odd digits were encoded at a different rate than even digits. However, in spite of the high significance level of this interaction, it accounted for less than $1 \%$ of the total sums of squares.

\section{Reanalysis of Early Studies}

Following the discovery that odd-odd digit pairs were responded to more slowly and with more errors than eveneven digit pairs, a literature search was undertaken to

Table 1

Mean Reaction Times (RT) and Standard Deviations (SD) for Young and Old Subjects for Even-Even and Odd-Odd Digit Pairs at Different SOAs

\begin{tabular}{|c|c|c|c|c|c|c|c|c|c|c|}
\hline \multirow[b]{3}{*}{ Digit Pair } & \multicolumn{10}{|c|}{ SOA (in msec) } \\
\hline & \multicolumn{2}{|c|}{0} & \multicolumn{2}{|c|}{250} & \multicolumn{2}{|c|}{500} & \multicolumn{2}{|c|}{750} & \multicolumn{2}{|c|}{1,000} \\
\hline & RT & $S D$ & RT & $S D$ & RT & $S D$ & $\mathrm{RT}$ & $S D$ & RT & $S D$ \\
\hline \multicolumn{11}{|c|}{ Young Subjects } \\
\hline Odd-Odd & 933 & 243 & 773 & 225 & 760 & 220 & 738 & 196 & 748 & 204 \\
\hline Even-Even & 724 & 153 & 591 & 125 & 575 & 125 & 582 & 155 & 585 & 121 \\
\hline \multicolumn{11}{|c|}{ Old Subjects } \\
\hline Odd-Odd & 1391 & 397 & 1181 & 416 & 1193 & 408 & 1137 & 342 & 1133 & 339 \\
\hline Even-Even & 980 & 230 & 823 & 202 & 790 & 195 & 787 & 191 & 808 & 197 \\
\hline
\end{tabular}


ascertain whether this phenomenon had been reported previously. No such reports could be found. However, reanalysis of data collected in early studies of the difficulty that children have in learning addition and subtraction problems did reveal such an effect.

Clapp (1924) studied third- and fourth-grade children's learning of the 100 possible addition problems that involve two single-digit numbers. He constructed a difficulty ranking for each problem on the basis of the number of errors children made while learning it. Clapp rank-ordered all 100 problems in terms of difficulty, with 100 being the most difficult and 1 the easiest. Since, in the experiments reported in the present paper, pairs involving either the digits 0 and 1 or the same digit (e.g., 4,4 or 7,7 ) were not used, such pairs were excluded from the analysis of the 100 problems that Clapp reported. These exclusions leave a corpus of 24 problems, 12 even-plus-even and 12 odd-plus-odd. The mean rank for these 12 even-pluseven problems was 57.6. The mean rank for the 12 oddplus-odd problems was 79.3. The difference is significant $[t(22)=2.72, p=.0119]$. It was felt that the use of a parametric $t$ test was justified here, since the ranking of each problem in Clapp's data was based on the average ranking of that problem for each of the students in the study. The $t$ test reported above, and all others in this paper, is two-tailed.

Clapp (1924) also examined 100 subtraction problems and 100 multiplication problems. The set of subtraction problems contained none in which the answer was less than zero. Because of this constraint, there were only six even-minus-even and six odd-minus-odd problems that used single digits other than zero and one, and that did not have the same digit on both sides of the minus sign. The mean rank of the odd-minus-odd problems was 34.8 , whereas that of the even-minus-even problems was 28.0. The difference is in the direction expected if odd digits are more difficult to process, but it does not reach significance $[t(10)=0.804]$. The set of multiplication problems contained all the single-digit by single-digit problems, so there were twelve even-by-even and twelve odd-by-odd problems that used different single digits. The mean difficulty rank for the even-by-even multiplication problems was 46.7 and that for the odd-by-odd problems was 55.4. Again, the difference is in the expected direction, but does not reach significance $[t(22)=1.192$, $p=.2448$ ]. Clapps's study included an analysis of 92 division problems, but it was not possible to analyze them for any odd versus even difference because only five used single digits other than zero or one.

For all three of the mathematical operations in which Clapp's (1924) data could be evaluated, the problems involving odd digits showed a higher ranked difficulty, with this effect reaching significance in the case of addition problems. Thus, Clapp's data support the hypothesis that odd digits are more difficult to process than even digits.

Knight and Behrens (1928) also undertook a study of the difficulty of the 100 single-digit addition and subtrac- tion problems. The subjects in Knight and Behrens' study were children in the second grade. Each of the addition and subtraction problems was given a "general difficulty rating" on the basis of the "number of responses to learn, number of errors encountered in learning [and] time of review responses" (p. 16). In addition, the time required for three "typical practice" trials on each problem and the number of errors made during learning were reported.

Significantly more errors were made on the 12 odd-plusodd than on the 12 even-plus-even problems [14.3\% vs. $7.1 \% ; t(22)=2.18 ; p=.0376$ ]. Practice time was significantly greater for the odd-plus-odd than for the evenplus-even problems $[4.75 \mathrm{sec}$ vs. $4.1 \mathrm{sec} ; t(22)=2.11$, $p=.0437]$. The mean difficulty rank of the odd-plusodd problems was higher than that of the even-plus-even problems [82.8 vs. $67.8 ; t(22)=2.66, p=.0135]$. The mean difficulty ranking of the six odd-minus-odd problems was 64.2 , whereas the mean ranking for the even-minuseven problems was 50.0 . This difference, although in the expected direction, did not reach significance $[t(10)=$ $1.86, p=.089]$. Overall, the results of the analysis of Knight and Behrens' (1928) data further confirm that children have greater difficulty with problems involving odd digits than with those involving even digits, with this effect being especially evident in the addition problems.

\section{EXPERIMENT 2}

Since the subjects in Experiment 1 had judged pairs of digits, the increased RT for odd-odd pairs could be due either to some inherent characteristics of the digits or to some factor that emerges only when two digits are judged. If the latter is correct, the "odd effect" should disappear when only a single digit is judged. In Experiment 2, this hypothesis was tested by having subjects classify a single digit as odd or even and measuring the time taken to do so.

\section{Method}

Subjects. The subjects were 20 Pace University undergraduates, 15 female and 5 male, who received extra credit in an introductory psychology course for their participation.

Apparatus and Procedure. The subjects sat approximately $50 \mathrm{~cm}$. in front of a closed-circuit television monitor used for stimulus presentation. Stimuli consisted of 100 individual digits, 50 odd and 50 even, presented in random order. Only the digits $2,3,4$, $5,6,7,8$, and 9 were used. A single digit appeared in the center of the screen and remained there until the subject responded. Digit or letter stimuli in this and all subsequent experiments measured $48 \times 65 \mathrm{~mm}$. Following the response, the subject was given feedback about his/her speed and accuracy. The RT in milliseconds appeared on the screen, and if the response had been incorrect, the word "ERROR" also appeared. The feedback remained on the screen for $500 \mathrm{msec}$. The screen then remained blank for a 1,300 msec intertrial interval. RTs greater than $1,000 \mathrm{msec}$ were discarded from the analysis. This resulted in only $0.1 \%$ of the responses being rejected.

The subjects responded by pressing one of two buttons on a handheld response box. For 11 of the subjects, the right-hand button was used to indicate an odd digit and the left-hand button was used to indicate an even digit. For the other 9 subjects, this pattern was 
reversed. The subjects were instructed to "go as fast as you can, but be as accurate as possible." All aspects of stimulus presentation and response recording were controlled by an Apple II computer

\section{Results and Discussion}

Preliminary analysis indicated that it made no difference whether the left or the right hand was used to indicate an even or an odd digit, so the data were collapsed over this variable. The results showed no difference between odd and even digits in the time needed to classify them (459 msec for odd digits and $454 \mathrm{msec}$ for even digits). However, more errors were made on odd (4.8\%) than on even $(3.7 \%)$ digits $[t(19)=2.252, p=.0345]$.

Thus, although the effect is small, odd digits are more difficult to classify than even digits. This result suggests that some inherent characteristic of the digits themselves is responsible, at least in part, for the effect observed in Experiment 1.

\section{EXPERIMENT 3}

The results of Experiment 2 show that the odd effect occurs even when a single digit is classified as odd or even, although the magnitude of the effect is reduced relative to that found in Experiment 1, in which two digits were classified. Experiment 3 was undertaken to examine further the generality of the odd effect by varying the type of judgment that subjects made about pairs of digits. In Experiment 3, subjects were required to distinguish between pairs of even and odd digits.

\section{Method}

Pairs of digits were presented centered on the screen with a space of $96 \mathrm{~mm}$ between them. If both digits in a pair were odd, the left button on the response box was to be pushed with the left thumb. If both digits were even, the right thumb was to be used to press the right button. If the digits in a pair were mixed, no response was to be made. The 18 subjects ( 8 female, 10 male) were Pace University undergraduates who received extra credit in their introductory psychology class for their participation. None had taken part in Experiment 2.

The fact that the odd response was mapped onto the left hand and the even response onto the right hand results, of course, in a confounding of response and responding hand. This is not a cause for concern, however, as handedness does not affect RT. Bradshaw and Perriment (1970), for example, reported a study of choice RTs used to examine laterality effects. They found no RT differences between the left and right hands. Umilta, Frost, and Hyman (1972) examined laterality effects in letter processing. All of their subjects were right handed and responded to letter stimuli presented to either the left or the right visual field with either their left or their right hands. In four separate analyses, there was never a significant difference in RT between the hands. The differences that were found were small (less than $11 \mathrm{msec}$ ) and averaged less than $1 \mathrm{msec}$; sometimes the left hand was a bit faster, other times the right hand was a bit faster. These differences reflect random error, not any systematic differences between the hands, even in a sample of right-handed subjects. Thus, the confounding here (and in Experiment 6) between responding hand and response presents no problem.
Temporal details of stimulus and feedback presentation were the same as in Experiment 2. Each digit pair was presented centered on the video monitor with six blank spaces between them. There were 34 pairs in which one digit was odd and one was even. In 17 of these pairs, the first digit was odd, the second even. The even digit was first in the remaining $17 \mathrm{mixed}$-digit pairs. There were 33 pairs in which both digits were even and 33 pairs in which both digits were odd. The order of pair type was random. RTs longer than $1,500 \mathrm{msec}$ were excluded from analysis. Only $1.8 \%$ of all responses were so excluded

\section{Results and Discussion}

The results showed that odd-odd pairs resulted in longer RTs than did even-even pairs [714 vs. $600 \mathrm{msec} ; t(17)$ $=4.497, p<.001]$. In addition, more errors were made to odd-odd pairs than to even-even pairs $[6.9 \%$ vs. $2.7 \%$; $t(17)=3.901, p<.001]$. These results show that the slowing of RTs for judgments about odd digits is also found in a two-digit classification task, as well as in a twodigit same/different task such as that used in Experiment 1 .

Thus, the results of Experiment 3 reveal that the odd effect is a robust one. It is little affected by the type of judgment (classification vs. same/different) that the subject is making. It is, however, attenuated when the subject is making a judgment about a single digit, as in Experiment 2 .

\section{EXPERIMENT 4}

The fact that the odd effect is, at the very least, greatly attenuated when only a single digit must be classified, as opposed to when two digits must be classified, suggests that the number of digits that appear in the stimulus set may be crucial. The effect of number of digits could work at least two ways. First, judging oddness may require some increment of extra time. This type of theory would predict that the difference between RTs to triples of odd and triples of even digits should be approximately $220 \mathrm{msec}$. This is based on the fact that in Experiment 2, in which a single digit was classified, the difference was $5 \mathrm{msec}$, and in Experiment 3, in which pairs of digits were classified, the difference was $114 \mathrm{msec}$. This prediction is based on the supposition that an additional "oddness detection" will be needed in the triple-digit case.

A second account of the odd effect is possible, though not especially plausible. There were two (an even number) digits in the digit pairs used for Experiments 1 and 3. Perhaps this evenness in the actual stimulus biased the subjects' responses toward even. In Experiment 2, only a single digit was classified and, thus, any bias would be toward odd. Although 1 is technically an odd digit, it may not be seen as being as typically odd as the other odd single digits, thus accounting for the relatively small difference in responding to odd and even digits in Experiment 2. Furthermore, the digit 1 never appeared as a stimulus in any of the experiments reported here. If the bias account is correct, there should be a reversal of the odd effect when digit triples are used because the odd number of 
stimuli will bias the subject toward the odd response. Experiment 4 tested these hypotheses.

\section{Method}

An additional digit was added to each digit pair used in Experiment 3 . The digit triples were presented centered on the screen with a space of $96 \mathrm{~mm}$ between each digit. This resulted in 48 digit triples in which all digits were odd, 47 in which all three were even, and 49 in which a mixture occurred. In none of the triples did any digit repeat, and the triples were constructed so that each digit appeared in each position an equal number of times, as far as was practicable. RTs in excess of $2,000 \mathrm{msec}$ were excluded from analysis. This resulted in an exclusion rate of $0.5 \%$. The subjects were 19 Pace University undergraduates, 9 females and 10 males. They received extra credit in their introductory psychology course for their participation. None had taken part in previous experiments in this series.

\section{Results and Discussion}

The results of Experiment 4 are inconsistent with both accounts of the odd effect mentioned above. Classification responses to digit triples containing three odd digits were slower than classification responses of digit triples containing three even digits [732 vs. $612 \mathrm{msec} ; t(18)=$ $8.09, p<.001$ ]. Also, there were slightly more errors made to odd than to even digit triples $(4.5 \%$ vs. $3.5 \%)$, but this effect was not significant $[t(18)=0.61]$.

The difference here between RTs to classify even and odd digit triples was $120 \mathrm{msec}$, almost the same as the difference in RTs between classification responses for even and odd digit pairs seen in Experiment 3, which was $114 \mathrm{msec}$. That the even-odd difference is so similar to that found in Experiment 3 with digit pairs argues that the additional digit did not require an extra, timeconsuming oddness detection. The fact that there was a large difference between even and odd digit triples also eliminates the hypothesis that the finding with digit pairs was due to a biasing effect of there being two digits in the stimulus.

\section{EXPERIMENT 5}

The results of the first four experiments reported here have been quite illuminating as to what the odd effect is not. However, they have not shed light on what causes the effect. Experiment 5 attempts to do so.

The words "odd" and "even" meet the criteria for a marked and an unmarked adjective, respectively. According to Greenberg (1966), an unmarked adjective has a higher frequency of usage than its marked companion. In accord with this criteria, "even" has a higher frequency in the English language than does "odd" (over 100 per million vs. 38 per million; Thorndike \& Lorge, 1944). Zimmer (1964) has proposed another criterion for distinguishing between the marked and the unmarked adjective in a pair. Negative prefixes cannot be attached to the marked member, but can be attached to the unmarked member. Thus, in the case of "odd" and "even," it is permissible to prefix "even" with a negative prefix (i.e., "uneven"), but this is not the case with "odd" ("unodd" is not a legal word).
That "odd" is a marked and "even" an unmarked adjective has considerable implications for the slowed RTs to judgments about odd digits demonstrated in the experiments described thus far. Evidence indicates that marked adjectives are more difficult to process than are unmarked adjectives. For example, Sherman (1976) found that sentences containing marked adjectives took longer to process, in a task in which subjects had to judge the "reasonableness" of sentences, than did sentences containing unmarked adjectives. If it is the case that the odd effect is due to "odd" being marked and "even" being unmarked, the same pattern of results should be obtained when subjects judge digit words as when they judge digits.

\section{Method}

The method was that of Experiment 2, with the exception that the digits used in Experiment 2 were replaced with the number names printed in upper-case letters. Each word was presented centered on the screen. The subjects were 26 Pace University undergraduates who received extra credit in their introductory psychology course for their participation. None of the subjects participated in any of the other experiments reported in this paper. One percent of trials were excluded from analysis due to RTs of greater than $1,200 \mathrm{msec}$.

\section{Results and Discussion}

The results were as predicted. The mean RT to classify the name of an odd number was $537 \mathrm{msec}$, whereas the mean RT for the classification of the name of an even number was $517 \mathrm{msec}$. This difference is significant $[t(25)$ $=2.71, p=.011]$. There were more errors made on odd digit names $(4.9 \%)$ than on even digit names $(4.0 \%)$, but this difference, although in the predicted direction, was not significant $[t(25)=1.44, p=.16]$. Thus, the results of Experiment 5 are consistent with the linguistic markedness hypothesis.

\section{EXPERIMENT 6}

It might be argued that the results presented so far in this paper are not due to the linguistic markedness of the "odd" concept but to something unique to the domain of digits and their names. The straightforward way of testing this possibility is to see if a similar pattern of results appears when subjects make judgments about nonnumerical stimuli that are or are not marked. If a similar pattern of results is obtained with nonnumerical stimuli as has been obtained so far with numerical stimuli, the argument that the odd effect is due to the marked nature of the odd concept will be correspondingly strengthened. The next three experiments examine this issue. What is needed is a pair of adjectives, one marked, the other not, that can be used unambiguously to describe common nouns. Such an adjective pair is "alive" and "dead."

\section{Method}

The method of Experiment 6 duplicated that of Experiment 3, with the major change that pairs of words were to be classified on the basis of whether both words were the name of something alive or the name of something dead. Word pairs were presented cen- 
tered on the screen, with $240 \mathrm{~mm}$ separating the words. If the two words on a given trial were names of something alive, the left button of the response box was to be pressed. If both words were the name of something dead, the right-hand button was to be pressed. If the pair was mixed (one word the name of something alive, the other the name of something dead), no response was to be made. Only eight different high-frequency words were used-the names of four living things (child, bird, tree, fish) and four nonliving things (desk, lamp, gold, wheel). The subjects were 18 Pace University undergraduates (10 female, 8 male) who received extra credit in their introductory psychology course for their participation. None had taken part in any of the other studies reported in this paper. RTs of more than 2,000 msec were excluded from analysis, with the result that $3.3 \%$ of trials were eliminated.

\section{Results and Discussion}

The results were as predicted. The mean RT for a correct response to an "alive-alive" word pair was $743 \mathrm{msec}$, whereas the mean RT to a "dead-dead" word pair was $848 \mathrm{msec}$. This difference is significant $[t(17)=$ $4.82, p<.001$ ]. More errors were made in the classification of dead-dead than alive-alive word pairs $(7.1 \%$ vs. $5.1 \%)$, but this difference failed to reach statistical significance $[t(17)=1.64, p=.116]$.

These results suggest that the underlying reason for the slower processing of odd as opposed to even digits in a pair is due to the fact that odd is an unmarked adjective and is therefore processed more slowly. However, one would have greater confidence in this explanation if further similarities could be demonstrated between the results found in the number-judgment experiments and in studies in which subjects made judgments about words. Experiments 7 and 8 were conducted with this in mind.

\section{EXPERIMENT 7}

This experiment was identical to Experiment 6, except that subjects made same/different judgments instead of classification judgments on pairs of words. That is, a "same" response was to be made if the items in a pair were both living or both dead, and a "different" response if the pair included a living item and a dead item.

\section{Method}

The word pairs were identical to those used in Experiment 6 . Elimination from analysis of RTs of greater than 2,000 msec caused $2.6 \%$ of responses to be eliminated. There were 25 subjects (15 female, 10 male), all Pace University undergraduates who received extra credit for their participation.

\section{Results and Discussion}

RTs to dead-dead pairs were slower than those to alivealive pairs $[1,076$ vs. $839 \mathrm{msec} ; t(24)=7.81, p<.001]$. Error rates were higher for dead-dead pairs $(23 \%)$ than for alive-alive pairs $(6 \%)$. This result was significant $[t(24)=7.81, p<.001]$. Mean RT for different responses was $1,027 \mathrm{msec}$. The error rate for different responses was $17 \%$. These results show that the slowing of judgments about dead-dead word pairs is not limited to a classification judgment, but generalizes to a same/ different judgment, a finding analogous to the results with judgments about digits.

\section{EXPERIMENT 8}

The effect of the odd or even identity of a digit was attenuated when subjects were required to make a classification of only a single digit (Experiment 2 ) or digit word (Experiment 5). Thus, for comparison, the subjects in Experiment 8 had to classify only a single word depending on whether it was the name of something alive or not.

\section{Method}

The subjects were 18 Pace University undergraduates, 4 male and 14 female, who received extra credit in their psychology course for their participation. None had participated in any of the other studies reported in this paper. The method of this experiment was identical to that of Experiment 2, with the obvious exception that single words, not single digits, were the stimuli. The eight words used in Experiment 6 replaced the digits 2 through 9. In the 100 total trials, there were 50 trials on which the word stimulus was the name of something living and 50 trials on which it was the name of something not living. The words were arranged in random order. RTs of greater than $1,200 \mathrm{msec}$ were eliminated from analysis, resulting in $0.3 \%$ of responses being eliminated.

\section{Results}

Making an alive versus dead classification of a single word took longer if the word was the name of something not living than if it was the name of something living [472 msec vs. $434 \mathrm{msec} ; t(17)=7.58, p<.001]$. The error rates were $4.8 \%$ for words that named something living and $4.5 \%$ for words that named nonliving objects. This difference is not significant $[t(17)=.391, p=.67]$.

The difference in classification times between words that were the name of something living and those that were the name of something not living was $38 \mathrm{msec}$. In Experiment 6 , in which pairs of words had to be classified, the difference was $105 \mathrm{msec}$. The data from both Experiment 6 and the present experiment were submitted to a two-way ANOVA with number of words classified as a between-subjects variable. The ANOVA revealed, not surprisingly, a main effect of word type [alive vs. dead; $F(1,34)=40.87, p<.001]$ and of the number of words to be classified [one vs. two; $F(1,34)=71.88, p<.001$ ] The interaction between the number of words to be classified and word type was also significant $[F(1,34)=9.15$, $p=.004]$. An ANOVA on the percent-error data from Experiments 6 and 8 revealed no significant effects.

\section{GENERAL DISCUSSION}

The first four experiments reported here demonstrate that under a variety of different tasks and types of judgments, even digits are processed faster and/or more accurately than odd digits. Experiment 5 shows that this effect $\alpha$ curs as well with the names of digits. The final three experiments show that a similar effect occurs with words 
that can be dichotomized on a concept other than oddeven-specifically, dead-alive.

The presence of the interaction between word type and number of words to be classified in the comparison between Experiments 6 and 8 heightens the similarity of the results obtained when digits are classified as odd or even and when words are classified as names of things living or nonliving. The slowed RT seen when pairs of odd digits are classified is eliminated when a single digit must be classified, but even digits still have an advantage in terms of a lower error rate. Similarly, the slowing seen when pairs of "not-living" words are classified is much reduced when a single word has to be classified. However, there was still significant slowing when single "not-living", words were classified, analogous to the slowing seen when single odd digit names were classified.

The slowing seen when single "not-living" words were judged may be due to a greater effect of markedness when explicitly linguistic stimuli are to be judged, as compared to the case in which symbolic stimuli such as digits are judged. That is, markedness may be a property more strongly associated with some internal linguistic representation(s) than with more conceptual, nonlinguistic representations such as digits, the latter being more strongly activated when actual digits are used as stimuli. I know of no direct test of this hypothesis. However, it does have the advantage of making a testable prediction. When pictures of living and nonliving things are judged, there should be a slowing for judgments about pairs of pictures of nonliving as opposed to living things, but this effect should be reduced for judgments about single pictures of nonliving things.

From a theoretical point of view, H. Clark (1973) has proposed that the marked versus unmarked distinction, as well as other characteristics of language, has its origin in the nature of the physical world as it is perceived by the child as language is being learned. $\mathrm{H}$. Clark (1973) pointed out that most of the spatial adjectives in English (e.g., long-short, far-near, high-low, etc.) are divided into pairs, one member being unmarked (e.g., long, far, high) and the other being marked (e.g., short, near, low). Both H. Clark and E. Clark reviewed evidence that children learn to use the unmarked member of markedunmarked adjective pairs before they learn to use the marked member.

Although there is undoubtedly a correspondence between the perceptual world that the language-learning child faces and the pattern of learning of marked and unmarked adjectives, the results of the present series of experiments demonstrate that an explanation of the marked versus unmarked distinction based on perceptual criteria alone is not adequate. One might argue that the marked- unmarked adjective pair "dead-alive" could be mapped onto some sort of spatial frame of reference because "dead" is in the past (i.e., "behind") and alive is at least in the present, and perhaps, in some sense, in the future (i.e., "in front"). However, it seems very unlikely that the odd versus even distinction could be mapped onto any type of spatial relationship in the same way.

\section{REFERENCES}

Bradshaw, J. L., \& Perriment, A. D. (1970). Laterality effects and choice reaction time in a unimanual two-finger task. Perception \& Psychophysics, 7, 185-188.

Cerella, J. (1985). Information processing rates in the elderly. Psychological Bulletin, 98, 67-83.

$\mathrm{C}_{\text {LAPP, }} \mathrm{F}$. (1924). The number combinations: Their relative difficulty and the frequency of their appearance in text-books (Bureau of Educational Research Bulletin No. 1). Madison: University of Wisconsin.

CLARK, E. (1973). What's in a word? On the child's acquisition of semantics in his first language. In T. Moore (Ed.), Cognitive development and the acquisition of language (pp. 65-110). New York: Academic Press.

ClaRK, H. (1973). Space, time, semantics and the child. In T. Moore (Ed.), Cognitive development and the acquisition of language (pp. 2763). New York: Academic Press.

Greenberg, J. (1966). Language universals. In T. Sebock (Ed.), Current trends in linguistics (Vol. 3, pp. 61-112). The Hague: Mouton.

Hines, T., \& Clauss, E. (1982, August). The odd-odd effect. Poster presented at the 90 th Annual Meeting of the American Psychological Association, Washington, DC.

Hines, T., Poon, L., Cerella, J., \& Fozard, J. (1982). Age-related differences in the time course of encoding. Experimental Aging Research, 8, 175-178.

KNIGHT, F., \& BEHRENS, M. (1928). The learning of the 100 addition and 100 subtraction combinations. New York: Longmans, Green.

KRUEGER, L. E. (1986). Why $2 \times 2=5$ looks so wrong: On the oddeven rule in product verification. Memory \& Cognition, 14, 141-149.

Krueger, L. E., \& HAllford, E. W. (1984). Why $2+2=5$ looks so wrong: On the odd-even rule in sum verification. Memory \& Cognition, 12, 171-180.

Posner, M, \& Boles, S. (1971). Components of attention. Psychological Review, 78, 391-408.

Shepard, R., Kilpatric, D., \& Cunningham, J. (1975). The internal representation of numbers. Cognitive Psychology, 7, 82-138.

SHERMAN, M. (1976). Adjectival negation and the comprehension of multiply negated sentences. Journal of Verbal Learning \& Verbal Behavior, 15, 143-157.

SudeVan, P., \& TAYlor, D. (1987). The cuing and priming of cognitive operations. Journal of Experimental Psychology: Human Perception \& Performance, 13, 89-103.

THORNDIKE, E., \& LORGE, I. (1944). The teacher's word book of 30,000 words. New York: Columbia University Teachers College.

Umilta, C., Frost, N., \& Hyman, R. (1972). Interhemispheric effects on choice reaction times to one-, two-, and three-letter displays. Journal of Experimental Psychology, 93, 198-204.

ZIMMER, K. (1964). Affixed negation in English and other languages: An investigation of restricted productivity. Word, 20(5, No. 2).

(Manuscript received February 20, 1987; revision accepted for publication June 2 , 1989.) 